\title{
Neutral Theory and Scale-Free Neural Dynamics
}

\author{
Matteo Martinello, Jorge Hidalgo, and Amos Maritan \\ Dipartimento di Fisica 'G. Galilei' and CNISM, INFN, Universitá di Padova, \\ Via Marzolo 8, 35131 Padova, Italy \\ Serena di Santo \\ Departamento de Electromagnetismo y Física de la Materia, \\ Universidad de Granada, 18071 Granada, Spain \\ and Dipartimento di Fisica e Scienza della Terra, Università di Parma, \\ via G.P. Usberti, 7/A-43124, Parma, Italy
}

Dietmar Plenz

Section on Critical Brain Dynamics, National Institute of Mental Health, Porter Neuroscience Research Center, 35 Convent Drive, Bethesda, Maryland 20892, USA

\begin{abstract}
Miguel A. Muñoz
Departamento de Electromagnetismo y Física de la Materia and Instituto Carlos I de Física Teórica y Computacional, Universidad de Granada, Avenida Fuentenueva s.n., 18071 Granada, Spain

(Received 21 April 2017; revised manuscript received 4 October 2017; published 26 December 2017)
\end{abstract}

Neural tissues have been consistently observed to be spontaneously active and to generate highly variable (scale-free distributed) outbursts of activity in vivo and in vitro. Understanding whether these heterogeneous patterns of activity stem from the underlying neural dynamics operating at the edge of a phase transition is a fascinating possibility, as criticality has been argued to entail many possible important functional advantages in biological computing systems. Here, we employ a well-accepted model for neural dynamics to elucidate an alternative scenario in which diverse neuronal avalanches, obeying scaling, can coexist simultaneously, even if the network operates in a regime far from the edge of any phase transition. We show that perturbations to the system state unfold dynamically according to a "neutral drift" (i.e., guided only by stochasticity) with respect to the background of endogenous spontaneous activity, and that such a neutral dynamics-akin to neutral theories of population genetics and of biogeography_implies marginal propagation of perturbations and scale-free distributed causal avalanches. We argue that causal information, not easily accessible to experiments, is essential to elucidate the nature and statistics of neural avalanches, and that neutral dynamics is likely to play an important role in the cortex functioning. We discuss the implications of these findings to design new empirical approaches to shed further light on how the brain processes and stores information.

DOI: 10.1103/PhysRevX.7.041071

\section{INTRODUCTION}

The introduction by Kimura in 1968 of the neutral theory-hypothesizing that most evolutionary change is the result of genetic drift acting on neutral alleles [1] caused much debate and a revolution in the way population genetics and molecular evolution were understood. In a similar endeavor, Hubbell proposed that most of the variability in complex ecological communities could be

\footnotetext{
*mamunoz@onsager.ugr.es
}

Published by the American Physical Society under the terms of the Creative Commons Attribution 4.0 International license. Further distribution of this work must maintain attribution to the author(s) and the published article's title, journal citation, and DOI.
Subject Areas: Biological Physics, Complex Systems, Statistical Physics ascribed to neutral dynamics of similar species which expand or decline as a result of stochasticity [2,3]. Neutral theories have in common that they neglect any a priori intrinsic difference between coexisting individuals, regardless of their "species" (allele, tree, etc.) type, implying that the dynamics is purely driven by random demographic effects. For instance, the introduction of a novel species within an established population triggers a random cascade of changes, or "avalanche," which-as a result of the implicit neutrality-does not have an inherent net tendency to either shrink or expand at the expenses of others. This marginal-propagation process generates scalefree avalanches, which resemble critical ones even if the system is not necessarily posed at the edge of a phase transition [3,4]. (See Appendix A for a brief mathematical summary of neutral theory and its statistical implications.) 
Neutral models have been successfully employed to explain the emergence of scale-free distributions in (i) epidemic outbreaks with neutral microbial strains [4], (ii) viral-like propagation of neutral memes [5], (iii) the evolution of the microbiome [6], and (iv) the renewal of the intestinal epithelium from neutral stem cells [7]. Is neutral theory susceptible to be applied to the neural dynamics of the brain? And, in particular, could it explain the emergence of scale-free neuronal avalanches that have been empirically reported for spontaneous neural activity?

The human brain has a special feature that is common to all mammalians: it is endogenously active; i.e., cascades of electrochemical activity at multiple time scales spontaneously pervade its dynamical state even in the absence of any apparent stimuli or task. Mounting evidence suggests that such an endogenous activity is not random, but structured, and it contributes significantly to stimulus-related responses, being essential to brain functioning. Specifically, spontaneous, spatiotemporal bursts of neural activity were reported to appear in the form of avalanches [8], whose sizes $s$ and durations $t$ are distributed as $P_{s}(s) \sim s^{-\tau} \mathcal{F}\left(s / s_{c}\right)$ and $P_{t}(t) \sim t^{-\alpha} \mathcal{G}\left(t / t_{c}\right)$, respectively, where $\tau \approx 3 / 2$ and $\alpha \approx 2$ are critical exponents similar to those of an unbiased branching process [9-11], $\mathcal{F}$ and $\mathcal{G}$ are scaling functions, and $s_{c}$ and $t_{c}$ are system-size-dependent cutoffs obeying finite-size scaling [12]. Similar results have been obtained both in vitro and in vivo, as well as for different tissues, preparation types, experimental techniques, and animal species (see, e.g., Refs. [13-19]). Remarkably, signs of scale invariance have been reported to vanish under abnormal circumstances such as under the influence of modified pharmacological conditions, under anesthesia, or in pathologies [20]. See Refs. [21-25] for overviews and discussions on the state of the art. Taken together, these observations suggest that scale-free avalanches are a generic feature of spontaneous activity in cortical tissues, suggesting that they stem from an underlying critical phenomenon (see, however, Refs. [26,27]). This conclusion seems to back the hypothesis that biological computing systems might operate at the edge of phase transitions [28-30], providing them with optimal transmission and storage of information, exquisite sensitivity to signals, and a number of other important functional advantages [31,32].

Scale-free distributed events or bursts of "activity" such as earthquakes, vortex avalanches in superconductors, and Barkhaussen noise are common place in nature (see, e.g., Refs. [33,34]) and are often ascribed to their underlying dynamics being poised at a critical point. The paradigm of "self-organized criticality" was developed to explain how and why natural systems could self-tune to the vicinity of critical points $[33,35,36]$; in this context, scale-free distributed avalanches turn out to be the fingerprint of critical points of a phase transition into quiescent (or "absorbing") states [37,38]. Despite the success and conceptual beauty of this framework, not all scale-invariant episodes of activity can be ascribed to underlying criticality [39,40]; for instance, power-law distributed excursion sizes and times can also emerge from unbiased random walks [41], selforganization to the edge of a discontinuous phase transition [42], the Yule-Simon or "the rich gets richer" process [40], and, as discussed above, neutral dynamics [4,7].

In this work, we explore the possibility and discuss the potential benefit that empirically observed neural avalanches could be scale-free as a result of an underlying neutral dynamics-i.e., that each single event of activity is indistinguishable from others and can potentially propagate through the network in a marginal way, i.e., without an intrinsic tendency to either expand or contractalternatively to being self-organized to the edge of a phase transition. That is, we explore whether scale-free avalanches could stem from the neutral competition of activity (generated from different sources or stimuli) for available space. Also, we show that causal avalanches are not necessarily approximated by experimentally measured avalanches commonly identified with a criterion of temporal proximity. We propose that the diversity encountered for causal avalanches with respect to their size, duration, as well as spatial and temporal realization provides a rich reservoir that the real neural systems could exploit for efficient coding, optimal transmission of information, and, thus, for memory and learning [43].

\section{RESULTS}

\section{A. Computational model and its phenomenology}

The leaky integrate-and-fire (IF) neuron represents one of the most successful approaches to model spiking activity in real neurons. Connections between IF neurons reflecting brain connectivity allow these neurons to linearly integrate inputs which-when reaching a threshold-make the neuron spike, initiating propagation of activity in the network and leading to a rich dynamical landscape. Recently, several models explored the propagation of avalanches of activity within this type of approach, using synaptic plasticity as a key regulatory mechanism [44-46]. In particular, the model by Millman et al. [44] was able to capture the empirical observation of bistability in cortical networks, i.e., the existence of two well-differentiated stable patterns of cortical activity, called up and down states (see, e.g., Refs. [47,48] and references therein, as well as Refs. [45,49], for related and rather interesting models). Briefly, the model of Millman et al. consists of $N$ leaky integrate-and-fire excitatory neurons forming a directed random Erdős-Rényi network with average connectivity $K$. Neurons integrate synaptic inputs from other neurons and fire action potentials, which rapidly deplete the existing synaptic resources. These resources recover at a slow time scale, thereby limiting the overall level of activity in the network (see Appendix B). The model can be tuned by controlling, e.g., its average synaptic strength. For weak synaptic strengths, a quiescent phase with very low levels 

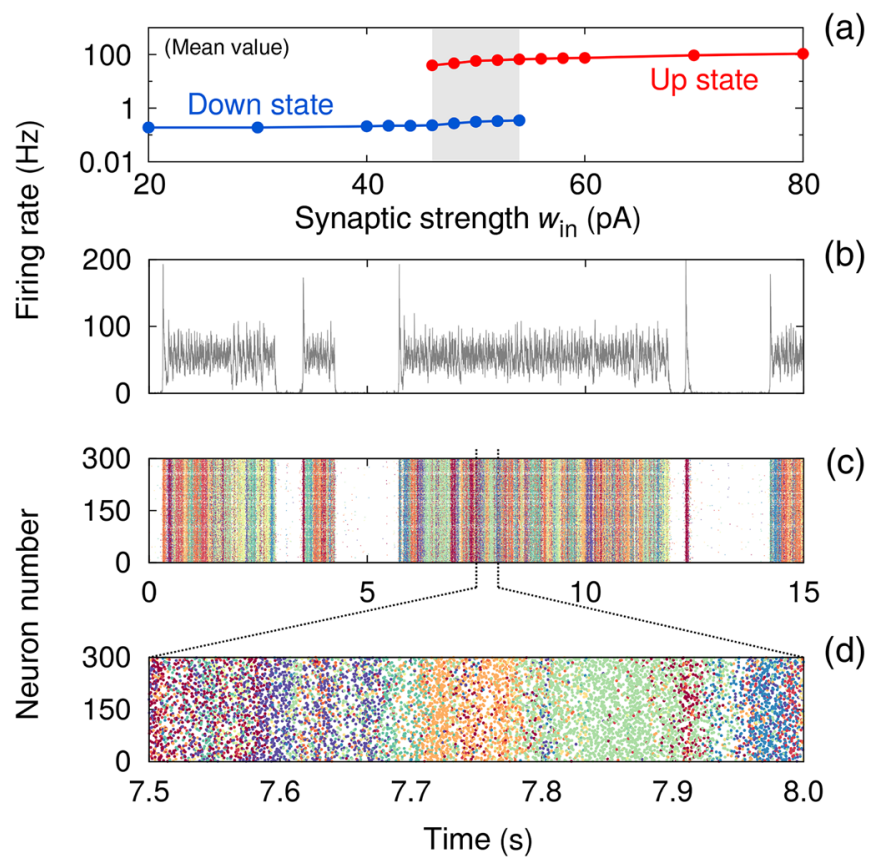

FIG. 1. Numerical integration of the model of Millman et al. [44] with $N=300$ neurons. (a) Phase diagram of the mean firing rate as a function of the synaptic strength parameter $w_{\text {in }}$. For low values of $w_{\text {in }}$, the stable state is a quiescent state with very low levels of activity (down state), whereas for large values of $w_{\text {in }}$, the system exhibits high levels of activity (up state). Both states coexist for intermediate strength values (shaded region), allowing for up-anddown transitions. Importantly, the transition is discontinuous. (b) Time series of the network firing rate for $w_{\text {in }}=50 \mathrm{pA}$ illustrate the system's bistability, with eventual (stochastic) jumps between up and down states. (c) Raster plot (for the same times as above) in which distinct colors are used for different causal avalanches. (d) Raster plot zoom (broken lines) demonstrating the intermingled and temporally overlapping organization of different causal avalanches. Model parameters have been set as in Ref. [44] (see Appendix B).

of activity, the down state, exists, whereas a second, stable state with high firing rates, the up state, emerges for large synaptic strengths [see Fig. 1(a)]. For intermediate strengths, spontaneous fluctuations allow for rapid up and down states alternations [see Fig. 1(b)]. This phenomenology_which could also be reproduced by keeping synaptic strength fixed and varying the synaptic recovery time or some other parameter of the model-corresponds to a discontinuous phase transition (see Fig. 1 herein and Fig. 1 in Ref. [44]) and therefore lacks the critical point characteristic of continuous transitions. However, remarkably, when tracking cascades of neuronal firing based on participating neurons, i.e., causal avalanches (see below), the model was shown to exhibit scale-free distributions of sizes and durations during up states, with associated exponents $\tau \approx 3 / 2$ and $\alpha \approx 2$, i.e., the hallmark of neuronal avalanches measured in brain activity. Accordingly, Millman et al. considered the up state as "self-organized critical," in contrast to the down state, which was "subcritical" with causal cascades that were not scale-free [44]. Given that critical dynamics emerge at continuous phase transitions, the presence of scale-invariant avalanches within the up state in the absence of any such transition in this model is unusual. This observation prompted us to identify possible alternative mechanisms for the emergence of scale-free avalanches within this and similar models.

\section{B. Causal avalanches}

Following Ref. [44], we tracked causal cascades or avalanches [50]. Each one is initiated when an external input depolarizes a neuron's membrane potential above threshold to fire an action potential. The activity propagates when the membrane potential of a postsynaptic neuron surpasses threshold. This threshold crossing results from the integration of synaptic input from active neurons in the network. Importantly, the postsynaptic neuron will become a member of the cascade that provided the last - and thus causal-input for the action potential being generated. This causality-based avalanche affiliation thus does not exclude subthreshold integration of input from many avalanches. Accordingly, a cascade stops when no threshold crossing can be linked to its past activity. The size of a cascade is the total number of action potentials triggered, while the cascade duration is the time span between its initiation and the time of its last action potential [44]. Avalanches were analyzed separately for up and down states in a network with $N=3000$ neurons, using different values of the external firing rate $f_{e}$; in particular, we analyzed the slow-driving case $f_{e} \rightarrow 0$ in which new cascades arrive at a slow pace.

Our results are in perfect agreement with the phenomenology found in Ref. [44]: cascades in the down state do not exhibit scale invariance but instead have a characteristic scale (see Fig. S1 of Supplemental Material [52]). On the other hand, cascades during up states distribute in size and duration according to power laws with exponent close to $\tau=3 / 2$ and $\alpha=2$, respectively [see Fig. 2(a)]. Also, the measured effective branching ratio is always close to unity; i.e., any tendency for increased propagation is counterbalanced by fewer recruitable sites (see Supplemental Material Fig. S2 [52]). As already observed in Ref. [44], these results are quite robust; they do not depend on how deep into the up state (i.e., how far from the transition point) simulations are run, nor on simulation details, nor do they change upon introducing inhibitory neurons (see Secs. S2 and S3 in Supplemental Material, respectively [52]). In particular, parameter values can be changed quite a lot, even away from neurophysiologically realistic ones, but as long as a stationary active or up state exists, causal avalanches within it unfold marginally, in a scale-free way.

\section{Time-correlated avalanches from time binning}

A key point of the previous analysis is that causal information between activation events (i.e., "who triggers whom") is essential to define avalanches. However, in 

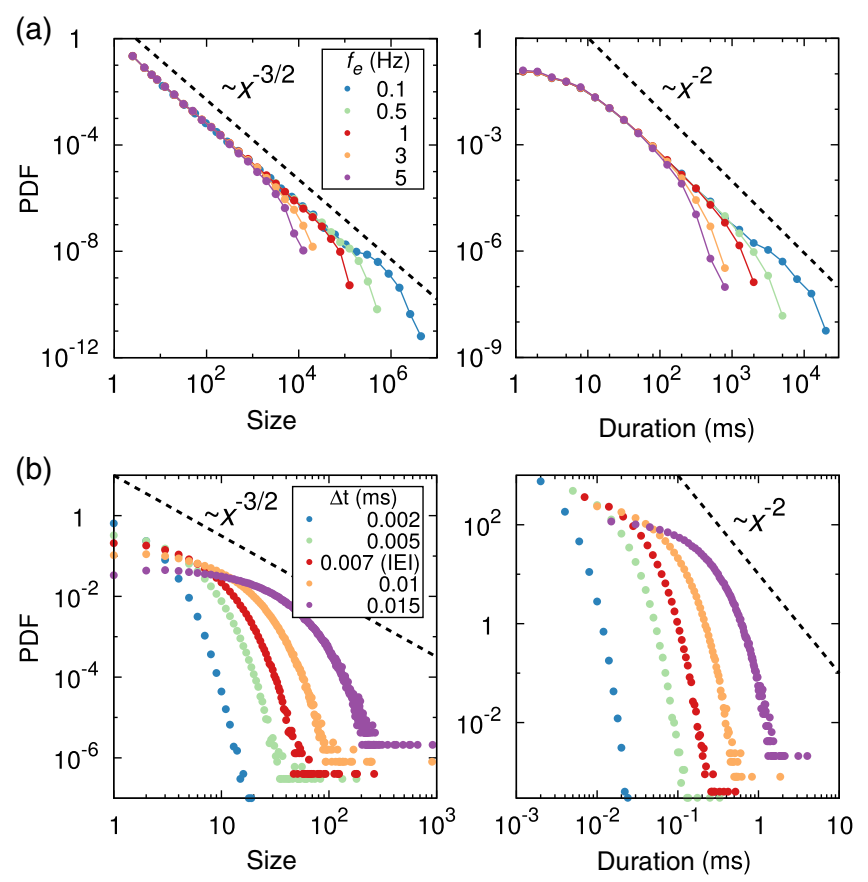

FIG. 2. Probability distribution functions (PDF) for the avalanche size and durations within the up-state phase in the model of Millman et al. [44] using two different methods (double logarithmic plot). (a) Causal avalanches were defined using the same criterion as in Ref. [44], for several values of the external input $f_{e}$, confirming the observation that sizes and durations are power-law distributed with the same exponents of an unbiased branching process, i.e., $\tau=3 / 2$ and $\alpha=2$, respectively [9,11]. (b) "Time-correlated" avalanches, defined with the standard temporal binning method [8] (which estimates causality by temporal proximity), using five different time intervals $\Delta t$ to bin the data, including one coinciding with the average interevent interval (IEI) as usually done in the analyses of empirical data [8], for $f_{e}=5 \mathrm{~Hz}$; in this case, distributions do not obey a power-law distribution but have a characteristic scale. In all cases, simulations were performed in a network of $N=3000$ neurons (model parameters as in Ref. [44]; see Appendix B).

empirical analyses it is not clear whether events occurring nearby in time-usually ascribed to the same avalanche in statistical analyses - are actually causally connected or not. The standard approach, which has been successfully used in the analysis of experimental data, where causal information of event propagation is typically not accessible $[8,13]$, consists in defining cascades from a series of discrete suprathreshold events, by choosing a discrete time bin $\Delta t$. An avalanche is defined as a sequence of successive time windows with at least one event in each that is preceded and ended by an empty bin. Following Refs. $[8,13]$, where it was shown that scaling relations obtained with different time bins $\Delta t$ could be collapsed, we take $\Delta t$ to be equal to the average interevent interval (IEI), defined as the average time interval between successive events [53]. Using this binning procedure in time series from the computational model, we find that cascade duration and size distributions obtained from up states are exponentially distributed with a characteristic scale, showing no signs of scale-invariant behavior [see Fig. 2(b)]. Distributions did not change qualitatively for different values of $\Delta t$. Thus, in the model of Millman et al., cascades based on temporal proximity differ significantly from cascades based on causal information. In the model of Millman et al., causal avalanches can (and do) coexist in time [see Figs. 1(c) and 1(d)]; thus, the temporal proximity approach does necessarily fail to uncover true causal avalanches. This finding is in contrast to the established scalefree avalanche distributions that emerge from experimental data based on temporal proximity.

Summing up, our observations, together with the lack of a continuous phase transition within this computational model, question the origin of scale invariance within up states and its actual relationship with empirically found (time-correlated) scale-free avalanches. To shed light on this problem, in the next section we analyze a minimal model which captures the main ingredients for activity propagation-but is more susceptible to mathematical analysis-showing that the observed scale-free causal avalanches in the basic approach as outlined in Millman et al. stem from an underlying neutral dynamics [4,7].

\section{Neutral (causal) avalanches in a minimal model for activity propagation}

In archetypical models of activity propagation such as the contact process, directed percolation, and the susceptible-infected-susceptible model [10,54], "active" sites propagate activity to their nearest neighbors or become deactivated at some transition rates. As a result, depending on rate values, there exist a quiescent and an active phase, as well as a critical point separating them [10,54]; avalanches triggered from a single initial event exhibit scale invariance only at criticality (see Fig. S4 of Supplemental Material [52]) and, if they are triggered at a sufficiently slow rate, they do not overlap.

In contrast, within the framework of neutral dynamics (that we are about to define), multiple avalanches can propagate simultaneously. The difference between critical and neutral avalanches can be vividly illustrated by considering a variant of the contact process, consisting of many different but equivalent "species" (or "labels" or "colors" or "types"). This model can be studied with parameters (rates) in the active phase, arbitrarily far from the phase transition, to explore the statistics of causal avalanches. More specifically, we consider a network with $N$ nodes that can be either active $(A)$ or inactive $(I)$. Here, we discuss the case of a fully connected architecture-for which mathematical analysis is simpler-but almost identical results are obtained for a directed random network, with the very same structure as employed in the model of Millman et al. (see Fig. S5 of Supplemental Material [52]). At every time, each single active site is assigned to a unique individual 
avalanche or species $k$ (the one from which it derives) and labeled by $A_{k}$. More specifically, the dynamics is as follows: (i) a new avalanche, with a new label, is initiated by the spontaneous activation of an inactive site at small driving rate $\epsilon$, (ii) active sites propagate the activity to neighboring inactive places at rate $\lambda$, and (iii) active sites become inactive at rate $\mu$. This is equivalent to the following set of reactions for $k=1, \ldots, M(t)$ :

$$
\begin{aligned}
I \stackrel{\epsilon}{\rightarrow} A_{M(t)+1}, \\
A_{k}+I \stackrel{\lambda}{\rightarrow} A_{k}+A_{k}, \\
A_{k} \stackrel{\stackrel{\mu}{\rightarrow} I,}{ } \text { I }
\end{aligned}
$$

where $M(t)$ is the total number of avalanches triggered up to time $t$. This dynamical process is neutral (or symmetrical) among species or avalanches as rates do not depend on label $k$ (see Appendix A for an extended presentation of neutral theories). The duration (size) of an avalanche $k$ is the time elapsed (total number of activations) between its spontaneous generation and the extinction of its label. Observe that different avalanches can coexist (all the more in the active phase, as in the model of Millman et al.) and that the total number of coexisting avalanches can vary in time. Also, their sizes are anticorrelated due to competition for a limited number of active sites (see Fig. S3 of Supplemental Material [52]). The state of the system is determined by $M(t)$ and the number of $k$-type active sites $n_{k}(t)$, or, equivalently, their corresponding densities $\rho_{k}(t)=n_{k}(t) / N$. The total density of active sites is defined as $\rho(t)=\sum_{k=1}^{M(t)} \rho_{k}(t)$. Importantly, just ignoring species labels, one realizes that the system of Eq. (1) is nothing but the standard contact process (with a nonvanishing rate for spontaneous activation $\epsilon$ ). Therefore, in the slow-driving limit $\epsilon \rightarrow 0$, the system exhibits a continuous phase transition for the total activity density at the critical point given by $\lambda_{c}=\mu[10,54]$.

We performed computer simulations of the dynamics described by Eq. (1) by means of the Gillespie algorithm [55] in a fully connected network of size $N=10^{4}$. Parameter values are chosen for the system to lie well inside the active phase, $\lambda=2, \mu=1$ (i.e., $\lambda=2 \lambda_{c}$ ), and $\epsilon$ taking small values such as $10^{-1}, 10^{-2}, 10^{-3}$, and $10^{-4}$. Typical time series for individual avalanches $\rho_{k}$, as well as for the total activity $\rho$, are depicted in Fig. 3(a).

Observe that the steady-state overall density (gray color) coincides, on average, with that of the contact process in the infinite size limit, $\rho^{*} \simeq 1-\mu / \lambda+\epsilon \mu /[\lambda(\lambda-\mu)]$ (see Appendix $\mathrm{C}$ for the derivation of this equality). In Fig. S2 of Supplemental Material [52] it is shown that, in full analogy with the more realistic model studied above, also in this simple model, the effective propagation rate of a given active site is equal to unity; i.e., the process is marginal in both cases. In particular, individual avalanches [colored curves in Fig. 3(a) experience wild fluctuations as a function of time. The statistics of avalanches is illustrated in Fig. 3(b), revealing that avalanche sizes and durations are power-law distributed
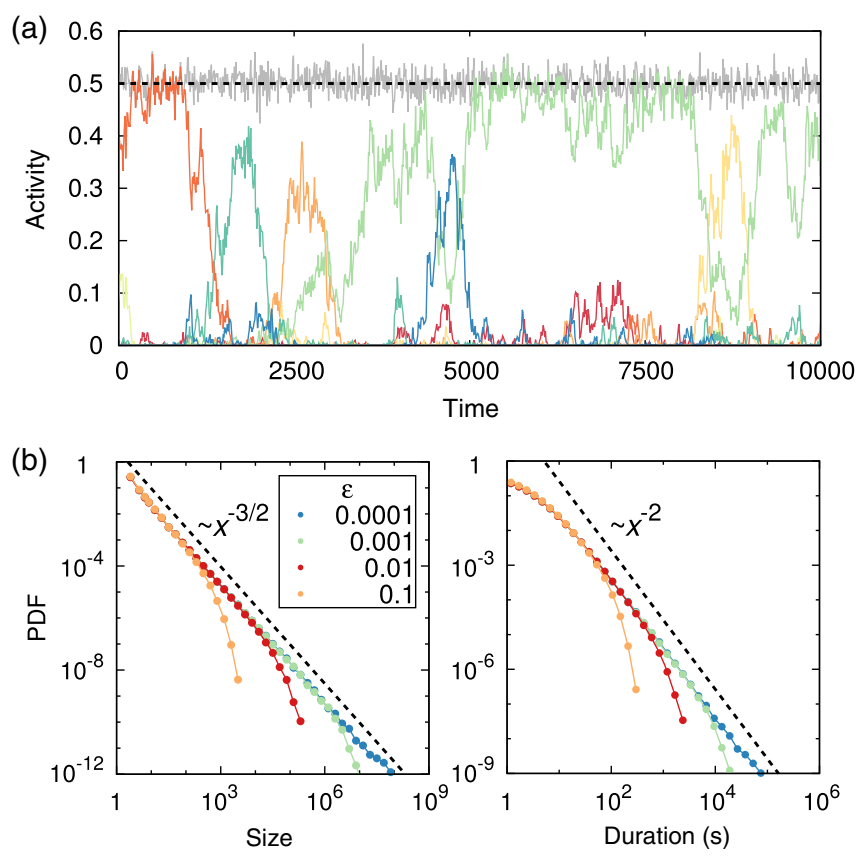

FIG. 3. Causal avalanches in a minimal model for propagation activity, defined as cascades of events initiated from the spontaneous activation of one unit, without overlap between avalanches (i.e., a given node cannot be simultaneously part of more than one avalanche). (a) The activity of each avalanche is defined as the density of active elements in the system belonging to that avalanche, identified with different colors in the plot. The global activity density is represented with the gray colored line. Parameters of the model are taken deep inside the active phase, $\lambda=2$, $\mu=1$, for a system size $N=10^{4}$ and small spontaneous activation rate $\epsilon=10^{-3}$. Whereas the global activity exhibits slight fluctuations around its steady-state value $\rho^{*} \simeq 1-\mu / \lambda$ (represented by the dashed line), individual avalanches can exhibit wild variability. (b) Probability distribution functions (PDF) for avalanche sizes and durations for different values of $\epsilon$ [other parameters as in (a), i.e., deep inside the active phase]. Avalanche statistics exhibit robust power-law scaling-limited by system size-with the same exponents of the neutral theory for avalanche propagation (marked with dashed lines for comparison).

with exponents $\tau=3 / 2$ and $\alpha=2$ in the limit of small spontaneous activation rate $\epsilon \rightarrow 0$. Remarkably, scale-free avalanches appear all across the active phase, $\lambda>\lambda_{c}$ (see Fig. S6 of Supplemental Material [52]).

To shed light on this result, we study analytically this simplified model in the large network-size limit. Starting from the master equation associated to Eq. (1), performing a system-size expansion for large but finite system sizes [56], the dynamics of a newly created avalanche is described by the following equation:

$\dot{\rho}_{k}=[\lambda(1-\rho)-\mu] \rho_{k}+\sqrt{\frac{1}{N}[\lambda(1-\rho)+\mu] \rho_{k}} \xi_{k}(t)$,

with the initial condition $\rho_{k}=1 / N$, and where $\xi_{k}(t)$ represents a zero-mean Gaussian white noise of unit 
variance (to be interpreted in the Itô sense [56]). If the system is very large, and when the rates lie within the active phase (i.e., $\lambda>\mu$ ), the total activity density exhibits very small fluctuations, remaining quite stable around the steady-state value, as illustrated by the gray colored time series in Fig. 3(a).

To understand the variability of individual avalanches, let us assume that in the steady state $\rho(t) \simeq \rho^{*}$, which introduced in Eq. (2) leads to

$$
\dot{\rho}_{k}=-\frac{\mu}{\lambda-\mu} \epsilon \rho_{k}+\sqrt{\frac{\mu}{N}\left(2-\frac{\epsilon}{\lambda-\mu}\right) \rho_{k}} \xi_{k}(t) .
$$

In the limit $\epsilon \rightarrow 0$, the deterministic or drift term in Eq. (3) vanishes, and the dynamics of avalanche $k$ can be simply written as

$$
\dot{\rho}_{k}=\sqrt{\rho_{k}} \xi_{k}(\hat{t})
$$

where, for simplicity in the notation, a factor $2 \mu / N$ has been reabsorbed into the time scale $\hat{t}$. Equation (4) represents a freely moving random walk with demographic fluctuations, which - as further discussed in Appendix A-describes the evolution of a species density in any neutral type of dynamics. In other words, once an avalanche starts, its statistics is entirely driven by neutral demographic fluctuations, without any net tendency to either expand or contract, regardless of the distance to the critical point [57]. Furthermore, the avalanche exponents associated with this neutral, noisedriven, dynamics are $\alpha=2$ and $\tau=3 / 2$; on the other hand, sizes and durations of time-correlated avalanches, as determined from time binning, are not power laws (see Fig. S7 of Supplemental Material [52]). These results hold all across the active (up) phase; on the other hand, in the quiescent (down) state, the steady-state activity $\rho^{*}$ goes to 0 , as the deterministic driving force in Eq. (2) is negative, leading to subcritical avalanches, as indeed reported in Ref. [44].

Thus, a simple approach allowed us to explicitly show that neutral dynamics among coexisting dynamically indistinguishable avalanches leads to scale-free distributions all across the active (up) phase, i.e., arbitrarily far away from the edge of the phase transition, where many different causal avalanches can simultaneously coexist, and with no relationship with self-organized criticality.

Our claim, relying on universality arguments, is that the same conclusion extends to the active phase of the model of Millman et al.; for such a case, detailed analytical calculations would be much more difficult to perform, but it seems rather plausible that the effective dynamics is also neutral as in the case of the simple model we discuss here, and that many neutral causal avalanches coexist, thus strongly supporting that as a matter of fact the scale-free causal avalanches in the model of Millman et al. stem from neutral dynamics and not from the model being self-organized to the critical point of any (nonexisting) continuous phase transition.

\section{CONCLUSIONS AND DISCUSSION}

A remarkable observation - that has elicited a great deal of interest-is that neural activity in the brain of mammals, including humans, occurs in the form of neuronal avalanches consisting of outbursts of neural activity intervened by periods of relative quiescence, across many resolution scales in a robust way [8,21]. For in vitro studies of relatively small networks, it seems plausible to assume that events occurring during one of such outbursts are causally connected, so that activity emerges at some location and transiently propagates through the network, causing a cascade of coactivations. However, there is no clear empirical validation that this is actually the case; diverse causally connected cascades could, in principle, occur simultaneously, hindering their experimental discrimination as individual avalanches. Obviously, the situation is much more involved in large neural networks as analyzed in vivo as diverse scales of resolution, e.g., from local field potential measurements, magnetoencephalography, functional magnetic resonance imaging, etc. There is no wellaccepted empirical procedure to actually disentangle causal influences, nor to discern whether different causal cascades of activations overlap (as they probably do in functional brains). Developing a protocol to fill such a gap is a task of utmost importance for the coming future (see Ref. [51]). In the absence of a better indicator, events of activity are customarily clustered together as individual avalanches, relying on a criterion of temporal proximity.

It remains to be fully elucidated what is the true nature of scale-free avalanches in actual neural systems. To shed light on this, here we scrutinize the most commonly referred model-introduced by Millman and coauthors [44] _ justifying the emergence of power-law distributed avalanches in networks of integrate-and-fire neurons with synaptic plasticity. First of all, we reproduced the findings in Ref. [44], and confirmed that the model exhibits two different phases in parameter space, an up state characterized by large average firing rates and a down one with small firing, separated by a discontinuous phase transition. We carefully analyzed the dynamics within the active phase, and corroborated that diverse avalanches can coexist, and that their sizes and durations are scale-free (with exponents, $3 / 2$ and 2, respectively) if and only if precise information on which neuron triggers the firing of whichwhich is accessible in computational models-is used to identify (causal) avalanches [51]. On the other hand, a different analysis - which is the one customarily applied to empirical data-based on defining avalanches through a time-binning procedure, blind to detailed causal information between activation events, does not reveal any trace of scale freedom in avalanche distributions. These observations naturally pose two important questions. First, if the 
analyzed model is not self-organized to the edge of a phase transition, where do the computationally reported scale-free (causal) avalanches within this model stem from? And second, does this model constitute a faithful representation of actual neural dynamics, including the experimentally observed scale-invariant avalanches?

To answer the first question we designed a simplified dynamical model with an overall phenomenology very similar to that of the model of Ref. [44]: i.e., it exhibits scale-invariant causal avalanches all along its active phase, regardless of the distance to a phase-transition point (which actually can be either a continuous or a discontinuous one depending on model details). This simplified model-a variant of the contact process with many different types of active sites-allows us to uncover that scale-invariant avalanches within the active phase stem from the neutral dynamics among diverse coexisting (causal) avalanches. In particular, if new seeds of activity are injected at a very slow rate in a system with recurrent background activity (i.e., in its active phase), each one does not have a net drift toward contracting or expanding in the background of recurrent activity in which it unfolds; its dynamics just follows demographic fluctuations, much as in neutral theories of population genetics. Moreover, the branching ratio is equal to unity and causal avalanches are power-law distributed-with the same exponents as an unbiased branching process-without the model being posed at the edge of a phase transition. In summary, the observed scale invariance in a well-accepted computational model for neuronal dynamics as well as in a simplified modeland in any other model for activity propagation with a stationary active or up state-stems from the neutrality or symmetry between diverse coexisting cascades of causally related events which coexists in a background of recurrent activity.

In regards to the second question above, it might occur that the discussed computational model does not reproduce all the phenomenology of actual neural dynamics in real networks. For instance, activity exhibits clear temporal clustering (so that measured power laws disappear when times are reshuffled [8]) and, as we have shown, this fact is mostly lacking in the model of Millman et al. This drawback was overcome in a more recent and detailed computational model including many additional neurophysiologically realistic ingredients (such as, e.g., inhibitory plasticity), which exhibits temporal clustering of activity together with scalefree avalanches [46]. In this case, avalanches are also measured employing causal information so that scale invariance is likely to stem from underlying neutrality, rather than criticality. It would be highly desirable to have a study of purely time-binned avalanches in this type of approach, allowing us to compare them with causal ones. For the sake of completeness, let us remark that recent work (analyzing a population of independent spiking units, all sharing a common time-correlated randomly changing firing rate) has illustrated the possibility-just opposite to the one discussed here- of having scale-free temporally defined avalanches, in the absence of causal ones (as neurons are not causally coupled) [27].

From a broader perspective, more complete computational models and/or analyses allowing us to scrutinize the possible emergence and interplay between neutrality and criticality are highly needed. In particular, relying on such models it should be possible to fully ascertain the relationship between neutral causal avalanches and empirically observed ones, but, for the time being, there remains a gap in the literature between causal avalanches in models and temporal correlated ones in experiments.

Finally, the main question that remains to be answered is, given that various types of functional advantages are ascribed to criticality, do these same advantages still exist if neuronal scale-free avalanches turn out to be the consequence of underlying neutrality rather than of the tuning to the edge of a phase transition? While we do not have a definite answer to this, we speculate that this type of power-law distributed coexisting causal avalanches could play a fundamental role in neural functioning. In particular, there are known biological mechanisms, such as learning rules, that take into account causal information (i.e., which neuron triggers the firing of which); a well-documented example is synaptic timing dependent plasticity (STDP) [43] by which synaptic weights are either reinforced or weakened depending on the relative spike timing between the pre- and postsynaptic neuron. STDP has been found to stabilize the dynamics of neural networks and to maintain reproducible patterns of causal neuronal avalanches [46]. Thus, patterns of activity, generated by neutral dynamics, and consisting on coexisting scale-free avalanches, could be stored and stabilized or "ingrained" by such a mechanism, allowing the network to spontaneously generate a large set of attractors and a broad dynamical repertoire, in a similar way in which cellular diversity-stemming from underlying neutral dynamic of stem cells-entails functional advantages in epithelial tissues [7]. These speculative ideas need to be much more carefully scrutinized, and we plan to do so in forthcoming work.

Summing up, some of the existing confusion surrounding different types of scale invariance in neural activity can be rationalized in the framework of neutral theories, posing new and fascinating questions that may contribute to clarify the criticality hypothesis in the cortex and its implications for function and learning.

\section{ACKNOWLEDGMENTS}

We are grateful to the Spanish-MINECO for financial support (under Grant No. FIS2013-43201-P; FEDER funds) and to P. Villegas, P. Moretti, S. Suweis, and S. Vassanelli for extremely useful discussions and comments. D. P. was supported by the Intramural Research Program of the NIMH (ZIAMH002797). 


\section{APPENDIX A: BRIEF SUMMARY OF NEUTRAL THEORY}

Consider a fully connected network with $N$ nodes (extensions to regular lattices or more complex networks architectures are also possible, but we stick here to the simplest case) and a number of possible states (be these species, alleles, opinions, etc.). The simplest neutraldynamic model is the voter model (VM) [10,58-60], also known as the Moran process in the context of population dynamics and population genetics (see, e.g., Ref. [61]). The VM assumes that there are two types of species that we call $A$ and $B$, respectively, and that the system or network is saturated, meaning that all nodes are always occupied, each one adopting one of the possible states at every time. The dynamics proceeds as follows: at each time step, one randomly chosen individual is "invaded" by a copy of another neighboring node at uniform rate, i.e., common to all the individuals in the population independently of their species labels. Without loss of generality, we first consider the case with just two species, and later on we explain how this can be employed to analyze the case with multiple species.

The so-defined, 2-species VM has been profusely studied in the mathematical literature; some of its main relevant features are [10,58-61] (i) it has no free parameters, (ii) it lacks any characteristic (length or time) scale and its dynamics exhibits scale invariance, and (iii) it is characterized by purely noise-driven diffusive dynamics (see Refs. $[59,60]$ for more mathematical in-depth presentations).

Now, we derive the coarse-grained mean-field description of a voter model; similar derivations can be found in the literature [61]. For the sake of illustration, let us consider also a more generic model in which $\lambda_{A}\left(\lambda_{B}\right)$ is the probability for $A(B)$ to invade a site in state $B(A)$, with $\lambda_{A} \neq \lambda_{B}$ in general; the VM dynamics is recovered imposing the symmetrical or neutral condition $\lambda_{A}=\lambda_{B}$.

As the system is saturated, the number of individuals for the other species is $n_{B}=N-n_{A}$ and the state of the system can be determined by the total number of individuals of $A$, $n_{A}$. The model can be expressed as a branching process [9], with transition rates $W\left(n_{A} \rightarrow n_{A}+1\right)=\lambda_{A} n_{B} n_{A} / N$ and $W\left(n_{A} \rightarrow n_{A}-1\right)=\lambda_{B} n_{A} n_{B} / N$. Using these rates, writing down the master equation for the probability of finding the system in a state $n_{A}$ at time $t$-or alternatively with a density of individuals $A, \rho_{A}=n_{A} / N$-and performing a standard large $N$ expansion, one readily obtains the following Fokker-Planck equation:

$$
\begin{aligned}
\frac{\partial P\left(\rho_{A}, t\right)}{\partial t}= & -\left(\lambda_{A}-\lambda_{B}\right) \frac{\partial}{\partial \rho_{A}}\left[\rho_{A}\left(1-\rho_{A}\right) P\left(\rho_{A}, t\right)\right] \\
& +\frac{\lambda_{A}+\lambda_{B}}{2 N} \frac{\partial^{2}}{\partial \rho_{A}^{2}}\left[\rho_{A}\left(1-\rho_{A}\right) P\left(\rho_{A}, t\right)\right],
\end{aligned}
$$

or its equivalent (Itö) Langevin equation

$$
\dot{\rho}_{A}=\left(\lambda_{A}-\lambda_{B}\right) \rho_{A}\left(1-\rho_{A}\right)+\sqrt{\frac{\lambda_{A}+\lambda_{B}}{N} \rho_{A}\left(1-\rho_{A}\right)} \eta(t),
$$

where $\eta$ is a zero-mean Gaussian white noise with $\left\langle\eta(t) \eta\left(t^{\prime}\right)\right\rangle=\delta\left(t-t^{\prime}\right)$. The neutrality condition $\lambda_{A}=\lambda_{B}$ implies that the deterministic drift in Eq. (A2) vanishes, thus $\left\langle\dot{\rho}_{A}\right\rangle=0$, i.e., the average density of each species remains constant on average; i.e., its population does not grow nor shrink on average, but it just experiences stochastic demographic changes as described by

$$
\dot{\rho}_{A}=\sqrt{\rho_{A}\left(1-\rho_{A}\right)} \eta(\tilde{t})
$$

where a factor $2 \lambda_{A} / N$ has been absorbed into the new time scale $\tilde{t}$.

Observe that this last equation describes a stochastic process (random walk) with two absorbing barriers at 0 and 1 , corresponding to either of the species $A$ or $B$, respectively, dominating the whole network. By neglecting the quadratic term in the noise (which is a valid approximation as far as the avalanche is small with respect to the much larger system size), the avalanche-time exponent $\alpha=2$ can be deduced from the first-passage time (return to the origin) statistics of this random-walk process, and using simple scaling arguments one can also easily derive $\tau=3 / 2$ for the avalanche-size distribution [62]; i.e., one recovers the mean-field exponents of the voter model (neutral theory) class (see, e.g., Ref. [4]). These power laws are truncated only by system size. The exponent values could also be analytically determined by employing the more standard generating function formalism for an unbiased branching process, as the rate of any cluster of $A$ nodes to expand $\lambda_{A} n_{A} n_{B} / N$ coincides with its rate to contract $\lambda_{B} n_{A} n_{B} / N$ in the neutral case $\lambda_{A}=\lambda_{B}$ (see, e.g., Refs. $[9,11]$ ).

Two apparently important differences between the VM dynamics and the multispecies contact-process-like one that we employ in the main text are (i) that many species appear in our model and only two of them in the VM and (ii) that in the VM the system is "saturated," in the sense that each single site is in one of the two possible states (opinions, alleles, species, labels, etc.) whereas in the model we study, some sites can be inactive, not belonging to any avalanche.

Regarding the first difference, for any given avalanche in the multispecies model, we can label it as " $A$ " and the rest of species labeled together as " $B$," which is feasible given that they all obey the same dynamical rules, i.e., are neutral. In respect to the second point, general principles of statistical physics, relying on universality, indicate that such a difference should have little effect on avalanche exponents. As a matter of fact, looking at the computational results for the dynamics of individual avalanches, such a difference is confirmed to be irrelevant. 


\section{APPENDIX B: MODEL FOR NEURAL DYNAMICS}

The model of Millman et al. [44] consists of a population of $N$ leaky integrate-and-fire excitatory neurons which are randomly connected in a directed graph to, on average, other $K$ neurons in the population (i.e., forming an Erdős-Rényi network [63]). External inputs $I_{e}^{k}(t)$ are Poisson distributed with rate $f_{e}$ and internal inputs $I_{\text {in }}^{k}(t)$ are generated from spiking neurons in the network ( $k$ accounts for the input number). Both internal and external currents are modeled by exponentials functions of amplitude $w_{e}$ in and characteristic time $\tau_{s}, I_{e / \mathrm{in}_{i}}^{k}(t)=w_{e / \text { in }} \exp \left[-\left(t-t_{s_{i}}^{k}\right) / \tau_{s}\right]$, where $t_{s_{i}}^{k}$ represents the corresponding spiking time of neuron $i$. Each individual neuron $i$ is described by a dynamical variable $V_{i}$ representing its membrane potential. When this value reaches a threshold value $\theta$, the neuron spikes and it may open - with probability $p_{r}$-each of its $n_{r}$ associated release sites per synapse, inducing a postsynaptic current. After spiking, the membrane potential is reset to the resting potential value $V_{r}$ for a refractory period $\tau_{r p}$, during which its dynamics is switched off. Synaptic depression is implemented by means of a dynamical "utility" variable $U_{i j}(t) \in[0,1]$ (for neuron $i$ and release site $j$ ), which modulates the release probability $p_{r} \rightarrow U_{i j} p_{r}$. The membrane potential obeys the following equation:

$$
\begin{aligned}
\dot{V}_{i}= & -\frac{V_{i}-V_{r}}{R C}+\sum_{k} \frac{I_{e_{i}}^{k}(t)}{C} \\
& +\frac{1}{C} \sum_{\substack{i^{\prime} \in n . n .(i) \\
j, k}} \Theta\left[p_{r} U_{i^{\prime} j}\left(t_{S_{i^{\prime}}}^{k}\right)-\zeta_{i^{\prime} j}^{k}\right] I_{\mathrm{in}_{i^{\prime}}}^{k}(t),
\end{aligned}
$$

where $R$ is the membrane resistance, $C$ its capacitance, $k$ is the spike number, $i^{\prime}$ runs over presynaptic neurons linking to $i$, and $j^{\prime}$ over its release sites; $\zeta_{i^{\prime} j^{\prime}}^{k}$ is a uniform random number in $[0,1]$ and $\Theta(x)$ the Heaviside step function. On the other hand, the synaptic utility $U_{i j}$ is set to 0 immediately after a release and recovers exponentially to 1 at constant rate $\tau_{R}$ :

$$
\dot{U}_{i j}=\frac{1-U_{i j}}{\tau_{R}}-\sum_{k} U_{i j} \Theta\left(p_{r}-\zeta_{i j}^{k}\right) \delta\left(t-t_{s_{i}}^{k}\right) .
$$

As Eqs. (B1) and (B2) are linear during successive events, they can be integrated exactly, which allows us to implement both synchronous (or clock-driven) and asynchronous (or event-driven) methods [64], leading to essentially indistinguishable results. When not specified, model parameters were taken as in Ref. [44]: $K=7.5, n_{r}=6, R=2 / 3 \mathrm{G} \Omega$, $C=30 \mathrm{pF}, V_{r}=-70 \mathrm{mV}, \theta=-50 \mathrm{mV}, w_{e}=95 \mathrm{pA}$, $w_{\text {in }}=50 \mathrm{pA}, \quad p_{r}=0.25, \tau_{r p}=1 \mathrm{~ms}, \tau_{s}=5 \mathrm{~ms}$, and $\tau_{R}=0.1 \mathrm{~s}$. We also studied versions of the model including inhibitory couplings, but this did not alter the main conclusions (see Sec. S2 in Supplemental Material [52]).

\section{APPENDIX C: TOTAL DENSITY OF ACTIVITY AT STATIONARITY IN THE MINIMAL MODEL}

Neglecting fluctuations from finite-size effects, the dynamics of the total density of activity for the process described by Eq. (1) becomes deterministic in the limit $N \rightarrow \infty$ :

$$
\dot{\rho}=[\lambda(1-\rho)-\mu] \rho+\epsilon(1-\rho),
$$

whose stationary solution $\dot{\rho}=0$ is

$$
\rho^{*}=\frac{\lambda-\mu-\epsilon+\sqrt{4 \epsilon \lambda+(\lambda-\mu-\epsilon)^{2}}}{2 \lambda} .
$$

Up to first order in $\epsilon$, Eq. (C2) can be written as

$$
\rho^{*} \simeq \begin{cases}\frac{\epsilon}{\mu-\lambda} & \text { if } \lambda<\mu \\ 1-\frac{\mu}{\lambda}+\epsilon \frac{\mu}{\lambda(\lambda-\mu)} & \text { if } \lambda>\mu .\end{cases}
$$

[1] M. Kimura, The Neutral Theory of Molecular Evolution (Cambridge University Press, Cambridge, England, 1984).

[2] S. P. Hubbell, The Unified Neutral Theory of Biodiversity and Biogeography (MPB-32) (Princeton University Press, Princeton, NJ, 2001), Vol. 32.

[3] S. Azaele, S. Suweis, J. Grilli, I. Volkov, J. R. Banavar, and A. Maritan, Statistical Mechanics of Ecological Systems: Neutral Theory and Beyond, Rev. Mod. Phys. 88, 035003 (2016).

[4] O. A. Pinto and M. A. Muñoz, Quasi-Neutral Theory of Epidemic Outbreaks, PLoS One 6, e21946 (2011).

[5] J. P. Gleeson, J. A. Ward, K. P. Oullivan, and W. T. Lee, Competition-Induced Criticality in a Model of Meme Popularity, Phys. Rev. Lett. 112, 048701 (2014).

[6] Q. Zeng, J. Sukumaran, S. Wu, and A. Rodrigo, Neutral Models of Microbiome Evolution, PLoS Comput. Biol. 11, e1004365 (2015).

[7] C. Lopez-Garcia, A. M. Klein, B. D. Simons, and D. J. Winton, Intestinal Stem Cell Replacement Follows a Pattern of Neutral Drift, Science 330, 822 (2010).

[8] J. M. Beggs and D. Plenz, Neuronal Avalanches in Neocortical Circuits, J. Neurosci. 23, 11167 (2003).

[9] T. E. Harris, The Theory of Branching Processes (Courier Corporation, Toronto, 2002).

[10] J. Marro and R. Dickman, Nonequilibrium Phase Transition in Lattice Models (Cambridge University Press, Cambridge, England, 1999).

[11] S. Zapperi, K. B. Lauritsen, and H. E. Stanley, SelfOrganized Branching Processes: Mean-Field Theory for Avalanches, Phys. Rev. Lett. 75, 4071 (1995).

[12] J. J. Binney, N. J. Dowrick, A. J. Fisher, and M. E. J. Newman, The Theory of Critical Phenomena (Oxford University Press, Oxford, England, 1993).

[13] T. Petermann, T. C. Thiagarajan, M. A. Lebedev, M. Nicolelis, D. R. Chialvo, and D. Plenz, Spontaneous 
Cortical Activity in Awake Monkeys Composed of Neuronal Avalanches, Proc. Natl. Acad. Sci. U.S.A. 106, 15921 (2009).

[14] A. Mazzoni, F. D. Broccard, E. Garcia-Perez, P. Bonifazi, M. E. Ruaro, and V. Torre, On the Dynamics of the Spontaneous Activity in Neuronal Networks, PLoS One 2, e439 (2007).

[15] A. Haimovici, E. Tagliazucchi, P. Balenzuela, and D. R. Chialvo, Brain Organization into Resting State Networks Emerges at Criticality on a Model of the Human Connectome, Phys. Rev. Lett. 110, 178101 (2013).

[16] E. Tagliazucchi, P. Balenzuela, D. Fraiman, and D. R. Chialvo, Criticality in Large-Scale Brain fMRI Dynamics Unveiled by a Novel Point Process Analysis, Front. Physiol. 3, 15 (2012).

[17] O. Shriki, J. Alstott, F. Carver, T. Holroyd, R. N. A. Henson, M. L. Smith, R. Coppola, E. Bullmore, and D. Plenz, Neuronal Avalanches in the Resting MEG of the Human Brain, J. Neurosci. 33, 7079 (2013).

[18] T. Bellay, A. Klaus, S. Seshadri, and D. Plenz, Irregular Spiking of Pyramidal Neurons Organizes as Scale-Invariant Neuronal Avalanches in the Awake State, eLife 4, e07224 (2015).

[19] S.-S. Poil, R. Hardstone, H. D. Mansvelder, and K. Linkenkaer-Hansen, Critical-State Dynamics of Avalanches and Oscillations Jointly Emerge from Balanced Excitation/ Inhibition in Neuronal Networks, J. Neurosci. 32, 9817 (2012).

[20] P. Massobrio, L. de Arcangelis, V. Pasquale, H. J. Jensen, and D. Plenz, Criticality as a Signature of Healthy Neural Systems, Front. Syst. Neurosci. 9, 22 (2015).

[21] H. G. Schuster, D. Plenz, and E. Niebur, Criticality in Neural Systems (John Wiley \& Sons, New York, 2014).

[22] D. R. Chialvo, Emergent Complex Neural Dynamics, Nat. Phys. 6, 744 (2010).

[23] D. Plenz, Neuronal Avalanches and Coherence Potentials, Eur. Phys. J. Spec. Top. 205, 259 (2012).

[24] J. M. Beggs, The Criticality Hypothesis: How Local Cortical Networks Might Optimize Information Processing, Phil. Trans. R. Soc. A 366, 329 (2008).

[25] L. De Arcangelis, F. Lombardi, and H. J. Herrmann, Criticality in the Brain, J. Stat. Mech. (2014) P03026.

[26] J. Touboul and A. Destexhe, Can Power-Law Scaling and Neuronal Avalanches Arise from Stochastic Dynamics?, PLoS One 5, e8982 (2010).

[27] J. Touboul and A. Destexhe, Power-Law Statistics and Universal Scaling in the Absence of Criticality, Phys. Rev. E 95, 012413 (2017).

[28] C. G. Langton, Computation at the Edge of Chaos: Phase Transitions and Emergent Computation, Physica (Amsterdam) 42D, 12 (1990).

[29] N. Bertschinger and T. Natschlager, Real-Time Computation at the Edge of Chaos in Recurrent Neural Networks, Neural Comput. 16, 1413 (2004).

[30] J. Hidalgo, J. Grilli, S. Suweis, M. A. Muñoz, J. R. Banavar, and A. Maritan, Information-Based Fitness and the Emergence of Criticality in Living Systems, Proc. Natl. Acad. Sci. U.S.A. 111, 10095 (2014).

[31] W. L. Shew and D. Plenz, The Functional Benefits of Criticality in the Cortex, Neurosci. 19, 88 (2013).
[32] O. Kinouchi and M. Copelli, Optimal Dynamical Range of Excitable Networks at Criticality, Nat. Phys. 2, 348 (2006).

[33] P. Bak, How Nature Works: The Science of Self-Organized Criticality (Copernicus, New York, 1996).

[34] J. P. Sethna, K. A. Dahmen, and C. R. Myers, Crackling Noise, Nature (London) 410, 242 (2001).

[35] H. J. Jensen, Self-Organized Criticality: Emergent Complex Behavior in Physical and Biological Systems (Cambridge University Press, Cambridge, England, 1998).

[36] G. Pruessner, Self-Organised Criticality: Theory, Models and Characterisation (Cambridge University Press, Cambridge, England, 2012).

[37] R. Dickman, M. A. Muñoz, A. Vespignani, and S. Zapperi, Paths to Self-Organized Criticality, Braz. J. Phys. 30, 27 (2000).

[38] J. A. Bonachela and M. A. Muñoz, Self-Organization without Conservation: True or Just Apparent ScaleInvariance?, J. Stat. Mech. (2009) P09009.

[39] G. Grinstein, Scale Invariance, Interfaces, and NonEquilibrium Dynamics (Springer, New York, 1995), pp. 261-293.

[40] M. E. J. Newman, Power laws, Pareto distributions and Zipf's law, Contemp. Phys. 46, 323 (2005).

[41] P. L. Krapivsky, S. Redner, and E. Ben-Naim, A Kinetic View of Statistical Physics (Cambridge University Press, Cambridge, England, 2010).

[42] S. di Santo, R. Burioni, A. Vezzani, and M. A. Muñoz, SelfOrganized Bistability Associated with First-Order Phase Transitions, Phys. Rev. Lett. 116, 240601 (2016).

[43] L. F. Abbott and S. B. Nelson, Synaptic Plasticity: Taming the Beast, Nat. Neurosci. 3, 1178 (2000).

[44] D. Millman, S. Mihalas, A. Kirkwood, and E. Niebur, SelfOrganized Criticality Occurs in Non-Conservative Neuronal Networks during 'Up' States, Nat. Phys. 6, 801 (2010).

[45] A. Levina, J. M. Herrmann, and T. Geisel, Dynamical Synapses Causing Self-Organized Criticality in Neural Networks, Nat. Phys. 3, 857 (2007).

[46] N. Stepp, D. Plenz, and N. Srinivasa, Synaptic Plasticity Enables Adaptive Self-Tuning Critical Networks, PLoS Comput. Biol. 11, e1004043 (2015).

[47] M. Steriade and I. Timofeev, Neuronal Plasticity in Thalamocortical Networks during Sleep and Waking Oscillations, Neuron 37, 563 (2003).

[48] J. Hidalgo, L. F. Seoane, J. M. Cortes, and M. A. Muñoz, Stochastic Amplification of Fluctuations in Cortical Upstates, PLoS One 7, e40710 (2012).

[49] A. Levina, J. M. Herrmann, and T. Geisel, Phase Transitions towards Criticality in a Neural System with Adaptive Interactions, Phys. Rev. Lett. 102, 118110 (2009).

[50] Here, we use indistinctly the terms "avalanche" and "cascade." See Ref. [51] for a recent and interesting analysis of causal avalanches on complex networks.

[51] R. V. Williams-Garcia, J. M. Beggs, and G. Ortiz, Unveiling Causal Activity of Complex Networks, arXiv:1603.05659.

[52] See Supplemental Material at http://link.aps.org/ supplemental/10.1103/PhysRevX.7.041071 for a set of additional analyses and figures.

[53] Even though the IEIs can vary for different experimental situations, size and duration distributions have been claimed to exhibit universal behavior with exponent $\tau \approx 3 / 2$ and 
$\alpha \approx 2$, respectively, provided that data are binned using the IEI.

[54] M. Henkel, H. Hinrichsen, and S. Lübeck, Non-Equilibrium Phase Transitions: Absorbing Phase Transitions, Theoretical and Mathematical Physics Vol. 1 (Springer, Berlin, 2008).

[55] D. T. Gillespie, Exact Stochastic Simulation of Coupled Chemical Reactions, J. Phys. Chem. 81, 2340 (1977).

[56] C. Gardiner, Stochastic Methods: A Handbook for the Natural and Social Sciences, Springer Series in Synergetics Vol. 25 (Springer, New York, 2009).

[57] Not surprisingly, Eq. (4) corresponds also to the mean-field description of an unbiased branching process [9].

[58] T. M. Liggett, Interacting Particle Systems, Classics in Mathematics Vol. 276 (Springer, New York, 2004).

[59] I. Dornic, H. Chaté, J. Chave, and H. Hinrichsen, Critical Coarsening without Surface Tension: The Universality Class of the Voter Model, Phys. Rev. Lett. 87, 045701 (2001).
[60] O. Al Hammal, H. Chaté, I. Dornic, and M. A. Muñoz, Langevin Description of Critical Phenomena with Two Symmetric Absorbing States, Phys. Rev. Lett. 94, 230601 (2005).

[61] R. A. Blythe and A. J. McKane, Stochastic Models of Evolution in Genetics, Ecology and Linguistics, J. Stat. Mech. (2007) P07018.

[62] S. di Santo, P. Villegas, R. Burioni, and M. A. Muñoz, Simple Unified View of Branching Process Statistics: Random Walks in Balanced Logarithmic Potentials, Phys. Rev. E 95, 032115 (2017).

[63] M. E. J. Newman, The Structure and Function of Complex Networks, SIAM Rev. 45, 167 (2003).

[64] R. Brette, M. Rudolph, T. Carnevale, M. Hines, D. Beeman, J. M. Bower, M. Diesmann, A. Morrison, P. H. Goodman, F. C. Harris, Jr. et al., Simulation of Networks of Spiking Neurons: A Review of Tools and Strategies, J. Comput. Neurosci. 23, 349 (2007). 